\title{
"AL PUEBLO EN PRIMER LUGAR, LE DEBO ESTA DEVOCIÓN; YO LOS QUIERO SALUDAR, CON TODA MI HERMANACIÓN": BAILES CHINOS EN LA VAGUADA DE LA RADIO LIMACHE, 1966-1969
}

\author{
"TO THE PEOPLE, IN FIST PLACE, I OWE THIS DEVOTION. I WANT TO SAY \\ HELLO WITH ALL MY TWINNING”: CHINESE DANCES AT THE COASTAL \\ TROUGH OF THE LIMACHE RADIO STATION, 1966-1969
}

Fernando Venegas Espinoza*

\begin{abstract}
En este artículo se analizarán los encuentros de bailes chinos del valle del Aconcagua que se realizaron entre 1966 y 1969 en el escenario de la Vaguada de la Radio Limache, en la región de Valparaíso. Lo que en dichas actividades se estaba conmemorando era el 12 de octubre, día de la raza, es decir, una festividad no religiosa, obligando a los alféreces y danzantes a expresarse dentro de ciertos formatos diferentes de los tradicionales, los del folklorismo, pero posibilitando al mismo tiempo la expresión de otras problemáticas relacionadas con lo que se conoce hoy como "canto a lo humano". Se observa además que, simultáneamente, los bailes chinos se vincularon a otros ámbitos no religiosos, como fue el caso de la Feria Internacional de Santiago de 1969 o reconocimientos de bailes chinos por parte de investigadores de la Universidad de Chile, lo que denota que en la época existió de parte de la cultura oficial una mayor valoración de la cultura popular. También se realiza un análisis al contenido de los cantos de los alféreces, permitiendo establecer algunas de las temáticas que se trataban durante estas actividades no religiosas.
\end{abstract}

Palabras claves: Bailes Chinos, canto a lo humano, cultura popular, Radio Limache.

In this article, the gatherings of Chinese dances of the Aconcagua Valley carried out between 1966 and 1969 at the trough of the Limache Radio Station scenario in the Valparaiso Region will be analyzed. What was being commemorated was the October 12, the day of the race. In other words, a non-religious festival, forcing the ensigns and dancers to express themselves within certain formats different from the traditional ones, those offolklorism, but at the same time facilitating the expression of other topics related to those which are known today as "singing to the human". It is also observed that simultaneously, the Chinese dances are linked to non-religious fields such the case of the Santiago International Fair in 1969 or the recognitions of a series of Chinese dances by researchers from the University of Chile. This denotes that in that time there was a greater appreciation of the popular culture by the official culture. A content analysis of the songs of the ensigns is also performed, allowed establishing some of the themes that were treated during these non-religious activities.

Key words: Chinese dances, singing to the human, popular culture, Limache Radio Station.

\section{Introducción}

Los encuentros de hermandades de bailes chinos parecen haberse realizado preferentemente en contextos de expresión o convergencia de la religiosidad popular, lo que ha llevado a que las investigaciones de las que han sido objeto hayan tenido como principal espacio de análisis las fiestas del calendario religioso. Los principales aspectos que se han resaltado han sido la incomprensión de la Iglesia católica frente a estas expresiones, además de su afán hegemónico en relación con las mismas, con las tensiones y resistencias que ha implicado desde los sujetos populares (Ruiz 1995; Mercado 2002, 2003; Villalobos 2012). También se ha realizado una caracterización de estas cofradías, buscando comprenderlas en profundidad desde la praxis, resaltando e intentando explicar la herencia indígena en la música y ritualidad (Ruiz 1995; Mercado 1995-1996; Mercado 2002; Mercado \& Rondón \& Piwonca, 2003; Mercado 2014; Godoy 2008; Venegas, 2010; Pérez de Arce, 2017). Otro aspecto analizado ha sido la tensión entre tradición y modernidad en la larga duración, pero sin dejar de poner atención en la historicidad de los sujetos que se están investigando (Contreras \& González, 2015). Destacan a su vez aquellos trabajos en donde se ha dado cuenta de manera genérica de la religiosidad popular (Salinas, 1991; Dannemann, 1988).

En este artículo intentaremos analizar los encuentros de bailes chinos a partir de su expresión en otros ámbitos. Se trata de los encuentros de

* Universidad de Concepción, Concepción, Chile. Correo electrónico: fervenegas@udec.cl 
hermandades que se realizaron entre 1966 y 1969 , en octubre, en el escenario de la Vaguada de la Radio Limache, en la región de Valparaíso. El motivo para congregarse fue la conmemoración de la efeméride del día de la raza, correspondiente al 12 de octubre. Es decir, una actividad no religiosa, tipo festival, que obligó -o permitió- a los alféreces y danzantes a desenvolverse en una modalidad de presentación diferente. Al no tratarse de una fiesta devocional, la tensión o confrontación entre Iglesia católica y religiosidad popular, típica de las fiestas religiosas tradicionales, perdió sentido. Como podremos ver, lo central no era "el canto a lo divino" sino el "canto a lo humano". Lo interesante es que, asociado a lo anterior, los bailes chinos comenzaron a ser convocados para presentarse en otros contextos no religiosos, desde actividades sociales locales hasta otras de connotación más amplia, como fue el caso de la Feria Internacional de Santiago de 1969. Por otra parte, una dimensión de estos bailes que parecía incomodar especialmente a la Iglesia católica, como también a los alféreces más jóvenes en las fiestas devocionales, era el carácter confrontacional que podían adquirir. En estos festivales, ese aspecto era particularmente importante, porque lo que se alentaba era precisamente la competencia entre cofradías. Gran parte del público asistente estuvo compuesto por los familiares, amigos y conocidos que debían cumplir esa función. No obstante, la organización aspiró a que la competencia se realizara en un ambiente de respeto y cordialidad.

Para problematizar lo que estamos planteando, en términos generales, tomaremos de Peter Burke la idea que el concepto de cultura popular no es homogéneo. De Mijaíl Bajtin, que la línea divisoria entre la cultura popular y de la elite no es infranqueable, y que, por tanto, es necesario poner atención en los puntos de convergencia. De Chartier, la importancia de analizar las apropiaciones culturales de los diferentes grupos más que encasillarlas como populares en oposición a una cultura de la elite o viceversa (Burke, 1991:17-32; Chartier, 1995:121138; Bajtin, 1989: 7-58).

En términos más específicos, resultan muy útiles los desarrollos teóricos que ha realizado Josep Martí respecto del folklorismo. El folklore es la disciplina académica que centra su preocupación en el estudio de la cultura tradicional, según perspectivas etnográficas, o bien, en la cultura tradicional propiamente tal. En tanto, si bien el concepto de folklorismo se relaciona con la definición de folklore, se trata de: "aquel conjunto de actitudes que implican una valoración socialmente positiva de este legado cultural que denominamos 'folklore"' (Martí, 1996: 82). Martí agrega que el folklorismo se compone de ideas y tendencias a la acción, es decir, "todas aquellas acciones motivadas por esta consciencia y estos sentimientos, como por ejemplo llevar a cabo proyectos conservacionistas o de divulgación de este legado tradicional, programar festivales de música tradicional, etc." (Ídem). En relación con su base ideacional, Martí destaca lo que define como el endofolklorismo, constituido por tres vertientes. $\mathrm{La}$ primera consiste en la vuelta hacia aquellos valores "que por su mayor primitivismo nos parecen más cerca de la hoy tan mitificada naturaleza" (Martí i Pérez, 1990, 323). La segunda, y la más relevante, es la ideología de cariz nacionalista que contiene. La tercera corresponde al tradicionalismo, es decir, "la presentación consciente e intencionada de contenidos culturales pertenecientes al pasado con la finalidad expresa de conservarlos y recuperarlos". De esta manera, mientras el folklore implica "continuidad", el folklorismo significa, "voluntad de continuidad" (Ibídem: 324). El concepto de folklorismo es pertinente para este trabajo. Como veremos, Hugo Arellano, propietario de la Radio Limache, al abrir un espacio para que los bailes chinos se expresaran en la Vaguada de su medio y difundirlo por las ondas del éter, tenía un propósito endofolklórico, en tanto le interesaba mostrar la cultura tradicional, pues obviamente, presentarla en un festival y ante una audiencia de radioescuchas potencialmente muy amplia, no era lo propio de estas cofradías danzantes.

Un estudio luminoso para esta investigación, por las relaciones comparativas que se pueden realizar, es el realizado por Brian Larkin (2008), referido a los medios de comunicación, infraestructura y cultura urbana en Nigeria, y más específicamente, de Kano, ciudad predominantemente musulmana situada en el norte de ese país. Larkin analizó críticamente la teoría de los medios, cuyo funcionamiento había sido explicado en sintonía perfecta con sus estructuras, y demostró que esta relación no se daba de ese modo, sino de manera bastante compleja. Junto con exponer cómo la radio y el cine contribuyeron a la articulación del esqueleto de la vida urbana de Nigeria, Larkin presentó la teoría de los medios en el contexto del mundo colonial, la que no fue lineal progresiva, sino intermitente, con ruidos y dificultades. En conjunto con los ferrocarriles y las 
autopistas, las radios y el cine fueron utilizadas por las autoridades británicas a partir de la década de 1930, para conformar un "tejido conectivo" que le permitiese vincular el territorio de Nigeria, y facilitar su gobernanza centralizada. Agrega que la radio y el cine también fueron tecnologías a partir de las cuales, implícitamente, se mostró la superioridad de Occidente. Del estudio de Larkin, lo que nos interesa es cómo evidencia que en la práctica, la agenda colonialista fue contradicha por la infraestructura de los medios. Primero, por problemas de carácter técnico: fallas de energía, transmisiones de frecuencia inestables, en síntesis, una frágil red de radiodifusión colonial. Segundo, porque lo que ocurrió fue que a los nigerianos se les abrió una oportunidad de experimentar la cultura más allá de lo local. En Kano, la radio no se escuchaba solo en las casas como en Europa, EE.UU. o México (Ornelas, 2014). Las autoridades instalaron altavoces de radio en las calles, edificios públicos, mercados, entre otros espacios comunes: se conectó el entorno urbano a corrientes de información globales y lo local también pudo proyectarse más allá de sus microespacios, pues los radioescuchas no fueron pasivos, sino que le dieron sus propios sentidos y significados a lo que recepcionaban. Por ello, las elites más conservadoras miraron esta tecnología con recelo. La discusión alcanzó incluso un carácter religioso. En síntesis, el uso de los medios de comunicación en Nigeria fue moldeado tanto por los objetivos del gobierno colonial como por las normas religiosas y culturales de la sociedad hausa.

Para el caso chileno, Patricio Manns realizó interesantes reflexiones acerca del significado del arribo de la radiotelefonía a Chile. Su hipótesis es que precisamente, fue utilizado por el Estado como una herramienta para desarrollar el nacionalismo, "poniéndolo al servicio de los mitos y apetitos de las clases detentadoras del poder" (Manns, 1986:41). Plantea que esto ocurrió mediante tres caminos. En primer lugar, del radioteatro histórico, en donde se resaltó las glorias del ejército chileno, por medio de "montajes vibrantes" que obtuvieron "gran audiencia". Pero en donde los héroes de ese ejército -en conflictos, por ejemplo, como la Guerra del Pacífico- eran de las clases dominantes. En segundo lugar, la difusión de música de aparente raíz campesina, como nacional. Manns indica que esto fue un mero artificio, porque Chile no era un país campesino. A su vez, la música urbana estaba notoriamente influenciada por el exterior, por intermedio de los conciertos, ópera, zarzuela, discos y emisiones radiales. Se recurrió entonces a la música campesina, la que se presentó como una veta que permitiría elaborar una música nacional (Ibídem: 41-42). A este formato musical y performativo que surgió en Chile a comienzos de la década de 1920, junto con el despliegue de la radiodifusión y la discografía, Rodrigo Torres (2004) le ha denominado "agronacionalismo". Se trató de conjuntos que se definieron y proyectaron como huasos, y que cantaron composiciones de contenido patriótico, además de "tonadas y cuecas, música típica campesina, casi siempre de tendencia paisajística y melancólica, en que se evoca y recrea con nostalgia al mundo rural, idealizándolo desde el punto de vista de la hacienda". Entre los conjuntos que destacaron estuvieron Los Huasos de Chincolco, Los Cuatro Huasos y Los Huasos Quincheros. Tuvieron una gran aceptación por los estratos altos de la sociedad, y los medios de comunicación y la industria musical los convirtieron "en un símbolo del folklore chileno y de la música campesina, aunque ninguno de sus músicos tuviera ese origen" (Subercaseaux, 2011: 216-217).

Un tercer elemento importante que propone Manns es el contexto en el que irrumpe la radiotelefonía, que es coincidente con el desarrollo de una conciencia trabajadora. Desde su punto de vista, la canción debía tener un papel desmovilizador, sería "un bálsamo que procurará ocultar, sino curar, las heridas sociales, que apartará a los trabajadores de sus ansias de reivindicación, y, que en lo que respecta al campesino, trabajará constantemente para donar una imagen paternal y bondadosa del terrateniente, que perpetuará a tradición de las 'familias sirvientes' que nacen, viven y mueren en las haciendas patriarcales" (Manns, 1986: 44).

La interpretación de Manns pudo estar muy influida por el contexto de época en que fue levantada, en que la dictadura cívico militar había desplegado un complejo mecanismo de control social en Chile. Al confrontarla con la propuesta de Larkin, aparece como demasiado rígida, en donde los medios de comunicación son solo una herramienta para el dominio de una clase sobre otra, por medio del Estado. En la práctica, sabemos que ello no fue así. Ejemplar en ese sentido es la experiencia de Violeta Parra. Más allá del trabajo de recopilación del folklore que ella realizó, fue notable su preocupación por la divulgación del mismo y su genial idea de colocar en los mismos 
medios de radiodifusión que estaban contribuyendo a la difuminación de la cultura tradicional, con el ingreso de la música extranjerizante, el folklore; aunque finalmente ello fuese una folklorización, sus esfuerzos por presentar tanto el canto a lo humano como lo divino, y dar a conocer los sones del guitarrón chileno, tratando de minimizar al máximo los procesos de edición, son encomiables. Esta iniciativa la impulsó por primera vez en Radio Chilena, y le valió incluso el Premio Caupolicán, en 1955 (Venegas 2017: 24, 29). Desde ese momento en adelante, Violeta grabaría muchísimas horas de radio. Solo en su estadía en la Universidad de Concepción, entre noviembre de 1957 y julio de 1958 registró por lo menos treinta. Precisamente, las radios universitarias fueron instancias relevantes de conexión de la cultura global con la local, como en contribuir en la difusión de otras perspectivas respecto de lo que oficialmente se denominaba como folklore, y como espacios de formación cívica. En el caso de la Radio de la Universidad de Concepción, inaugurada oficialmente en 1959, su rector, David Stitchkin resaltó el impacto que esperaba tuviese en la población adulta, principalmente la que se encontraba en los campos, "al margen o con difícil acceso a los servicios que prestan las instituciones culturales" (Ibídem: 84)

En el contexto señalado es que profundizaremos en la experiencia propiciada desde una radio local, en Limache, en la segunda mitad de la década de 1960. Comprobaremos que tal como lo planteó Larkin respecto de Kano, las posibilidades de acción mediante la radiodifusión fueron múltiples y complejas, y no estuvieron supeditadas solo a los controles o pautas editoriales de los gobiernos de turno.

La hipótesis con la que trabajaremos es que la acogida que tuvo esta actividad tanto entre las hermandades como el público común, se podría explicar porque se expresó en un momento en que la cultura popular estaba en una etapa de despliegue y valoración social ascendente, por lo que los festivales y su transmisión radial fueron uno de los canales por donde esta va a comenzar a fluir.

\section{Metodología}

Este estudio ha sido construido a partir de la confrontación de tres tipos de fuentes. En primer lugar, a partir de un trabajo de recopilación de los cantos y danzas de la cofradías de bailes chinos que se presentaron en Limache, realizado por Enrique
Bello y la fotógrafa Rebeca Yáñez, que fue publicado por la Revista Educación, en octubre de 1968. Analizaremos sus contenidos, constatando que en ellos "lo humano" es lo esencial, valorándose las posibilidades del encuentro, el rol de la radio como medio de comunicación, las problemáticas de las que están dando cuenta, como era la sequía de esos años, dejando espacio para referirse a la contingencia. Se evidencia además que, por lo menos en algunos de estos campesinos, había una conciencia crítica respecto de los verdaderos intereses de la clase política, en contraposición a la perspectiva tradicional, que ha considerado al mundo rural como políticamente inactivo (Bauer, 1994: 257-278). En segundo lugar, esta documentación fue complementada con la revisión de prensa local y regional correspondiente a los años que se realizaron los encuentros de bailes chinos. A su vez, también se confrontó lo revisado con el material que Etnomedia masterizó y editó en formato $\mathrm{CD}$ y que está citado en la bibliografía. En tercer lugar, destacamos la incorporación de los testimonios orales, tanto para reconstruir el contexto sociohistórico en el que comenzó a funcionar la radio Limache como para las razones que explican la iniciativa. Contamos con dos entrevistas. La primera de ellas corresponde a un destacado alférez, Faustino Morales. Fue una conversación sostenida el 2002, en el marco de una iniciativa personal de rescatar la memoria de Limache, fue con un carácter abierto y privilegiando recoger la trayectoria de vida. Se registró en casete y solo se transcribió con motivo de este estudio, 12 años después de efectuada. En el caso de la entrevista a Hugo Arellano se hizo en el marco de una investigación pertinente al asociacionismo y la sociabilidad, el 2010. También fue una entrevista abierta. En ambas situaciones se preguntó expresamente por la problemática que es materia de esta investigación. En el caso de Hugo Arellano, fue exhibiéndole el reportaje que realizó la Revista de Educación. Lamentablemente don Hugo no recordó mucho. Además, todo el material que había en su radio se perdió cuando fue destruida en septiembre de 1973, con motivo del golpe de Estado. El análisis que realizaremos de la documentación señalada será esencialmente de carácter cualitativo y con un enfoque microhistórico.

\section{Contexto espacial y cultural}

Los festivales de bailes chinos fueron organizados por el comunicador social Hugo Arellano 
Herrera. Emigrante de Valparaíso, en donde había trabajado en radios y como periodista, además de desempeñarse como director del diario La Unión, llegó para instalarse en Limache y establecer la radio de nombre homónimo. Para dimensionar el significado de su migración laboral, considérese que, según el Censo de 1960, Valparaíso sumaba 255.356 habitantes, mientras Limache, solo contaba con 19.638, con un importante porcentaje de población de carácter rural (Dirección de Estadísticas y Censos, 1960).

Arellano, de militancia socialista, indicó que se sintió profundamente impactado por la realidad de la comarca a la que arribó, a la que consideró significativamente atrasada respecto del centro urbano del que provenía, además de controlada desde dos haciendas y por un puñado de comerciantes, conocidos como "los doce apóstoles" (entrevista a Hugo Arellano, Limache, 2010).

A partir de su diagnóstico, Arellano asumió una posición crítica desde su radio, que le valió el reconocimiento de los sectores populares, tanto de la zona urbana como rural, quienes apreciaron su medio como una plataforma para denunciar abusos. La valoración de obreros y campesinos llevó a que fuese elegido como regidor, llegando a ser la autoridad más importante en tiempos de la Unidad Popular. Su radio sería bombardeada y destruida por los marinos cuando se inició el golpe el 11 de septiembre de 1973 (Arellano 2010).

El área de la comuna de Limache, y muy especialmente la zona de Olmué (que se elevaría a la categoría de comuna en 1966), era reconocida como una "zona huasa", representativa de la cultura del Chile tradicional. Parte de esa cultura era la que se expresaba en los llamados bailes chinos, que se reunían en torno a ciertas festividades religiosas en Caicai y Las Palmas (Contreras \& González, 2009; Venegas 2009; 2014). Enrique Bello lo sintetiza de manera más panorámica para fines de la década de 1960:

"Solo en las provincias de Aconcagua y Valparaíso y en todo el Norte Grande los 'chinos' han permanecido. En dichas dos provincias continúan funcionando para determinadas festividades católicas, como las del Corpus Christi, el baile chino de Puchuncaví, la Virgen de Petorquita y Pachacamita, la Virgen del Rosario de Valle Hermoso en La Ligua, la fiesta de la
Cruz de Mayo que, cuando éramos niños, conocimos en pleno campo sureño... vigente aun en los Maitenes de Puchuncaví, Tabolango y Boco; la Virgen de Lourdes de Caicai y el Niño Dios de las Palmas en Quebrada Alvarado, ambas en Limache. La zona entera está folclorizada con danzantes y poetas. Hemos visto hasta en el estiradísimo Zapallar danzas de chinos de una cofradía constituida casi totalmente por pescadores" (Bello y Yáñez 1968:46-47)

\section{Bailes chinos en la Vaguada de la Radio Limache}

No es casual que por la sintonía y cercanía que estableció Hugo Arellano con los sectores populares, haya dado una valoración significativa a los bailes chinos. En una entrevista realizada en 1968, explicó sus razones para impulsarlos: "Se trata de que renazca el folklore. No olvidemos que Limache es el límite de la zona en que todavía existe el verdadero folklore chileno. Más al sur no existen manifestaciones de una cultura en la que influyen los quechuas y los incas. Y es decepcionante como en las ramadas dieciocheras se escuchan discos go-go y ritmos extranjeros, mientras se olvida completamente nuestra música"; y precisó: "Es indudable la gran influencia incásica y quechua en las danzas. No olvidemos que Limache es el límite de estas costumbres; más al sur, el folklore es diferente" (Revista 7 Días, octubre de 1968).

El endofolklorismo de Arellano se manifestó en que además invitó a especialistas de la Universidad de Chile a que asistiesen a la actividad, poniendo a su disposición las grabaciones realizadas para posibilitar las transcripciones de las décimas de los alféreces. Este fue el caso de María Ester Grebe, profesora de música de la Facultad de Ciencias y Artes Musicales de la Universidad de Chile, que en 1968 asistió al encuentro junto con el profesor y musicólogo soviético Arkadio Viatkin. En esa oportunidad, Grebe señaló:

"Las danzas constituyen una expresión folklórica ritual de función ceremonial; su valor se basa en una larga trayectoria histórica. Originarias de España, fueron recibiendo la influencia indígena. Los vestuarios y ritmos provienen, probablemente, de la época precolombina" (Ídem). 


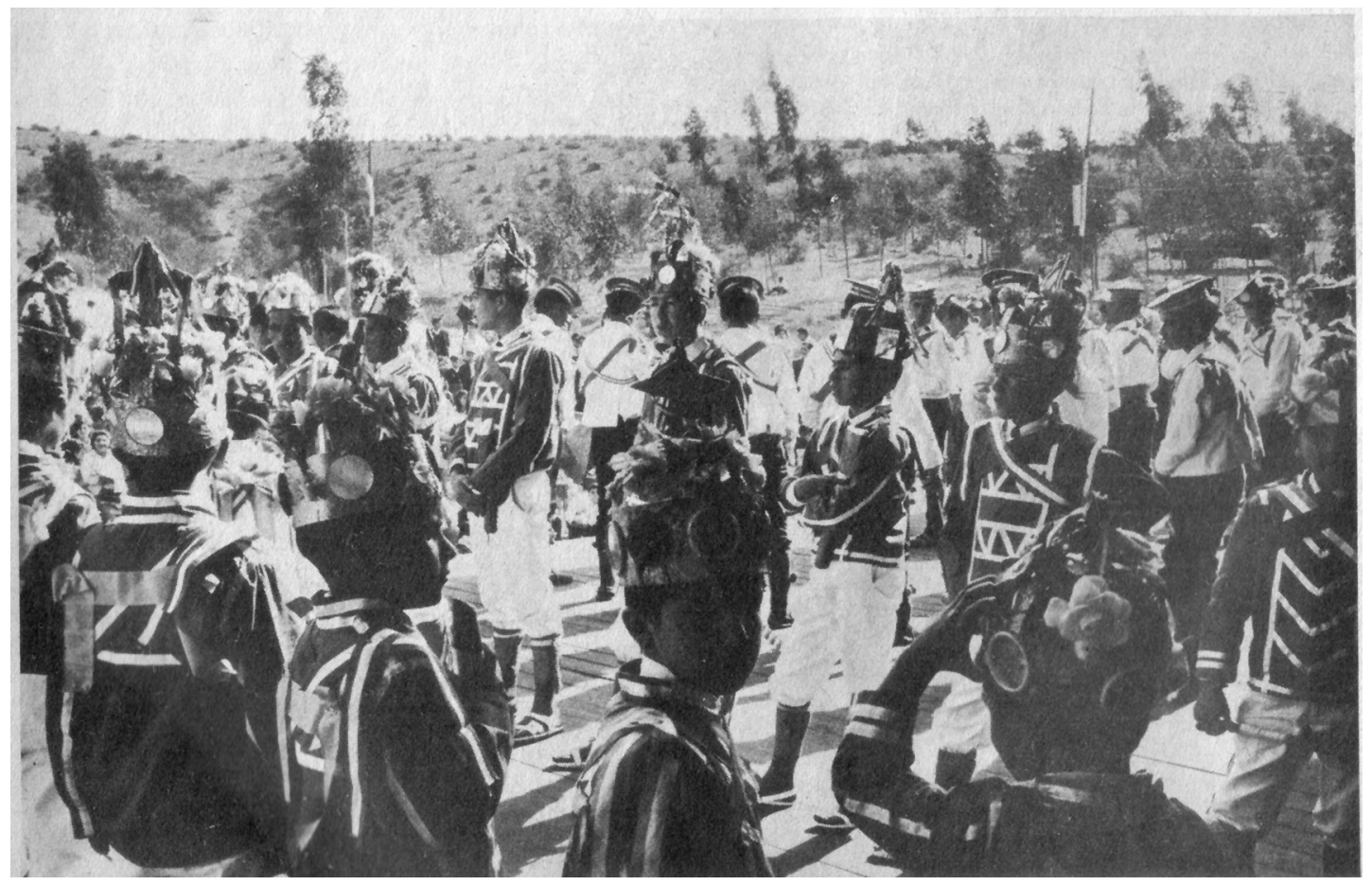

Fotografía 1. En ella se aprecian los conjuntos esperando se les llame a formar para comenzar los bailes. Se observan tanto niños como jóvenes y adultos. Enrique Bello apunta que "algunos de estos niños dan prueba de verdadera precisión rítmica y oído musical”. Fotografía de Rebeca Yáñez (Bello y Yáñez, 1968:44).

Grebe concurrió al menos a tres de los encuentros, en los que se conservó en registro de soporte magnetofónico y fotográfico, materiales que se encuentran en el fondo homónimo dependiente del Departamento de Antropología de la Universidad de Chile (Contreras \& González \& Pineda, 2014 a y 2014 b, 2014 c).

En ese sentido, y como de alguna manera lo sugiere el mismo Enrique Bello, Hugo Arellano es representativo del creciente interés por "rescatar el folklore" que comenzó a tomar fuerza en Chile a partir de la década de 1920, con el trabajo de los músicos Pedro Humberto Allende, Carlos Lavín, Carlos Isamitt y Filomena Salas. Posteriormente aparecerían en escena Margot Loyola y Violeta Parra.

Del primer encuentro de bailes chinos de 1966 encontramos escasas referencias. Solo las noticias que anuncian el evento, pero ningún apunte acerca de su convocatoria y trayectoria.

En su segunda versión, octubre de 1967, se anunció la participación de varias agrupaciones danzantes con sus respectivos alféreces: baile de la Virgen del Carmen de Petorquita, con su alférez Luis Tapia Pimentel; baile de la Cruz de Mayo de
Tabolango, con su alférez Arturo Ogaz; baile del Niño Dios de Las Palmas, alférez Carlos Bernales Torres; baile de Los Maitenes, alférez Faustino Morales; baile del Granizo, alférez Luis Araos Delgado; baile de Valle Hermoso, alférez Roberto Páez; baile del Olivo, de Boco, alférez Héctor Morales; y bailes de Santa Marta de Longotoma y de la Virgen de Pachacamita, de los cuales no se especificó sus alféreces. Al igual que en su primera versión, solo contamos con información previa a la actividad que aporta una idea general de lo planificado, aunque con un mayor desarrollo. Se indica que comenzaría a las 10:00 AM del día 12 de octubre. A esa hora, los bailes, reunidos todos en la estación de Limache, "se dirigirían bailando sus coreografías autóctonas y recitando las plegarias que les son propias, hacia 'La Vaguada', donde tendrá lugar el evento" (El Mercurio, Valparaíso, 10 y 12 de octubre de 1967). Para tener una idea más concreta de lo que se está informando, debe considerarse que desde la estación, situada en el barrio de San Francisco, hasta la radio Limache, emplazada a la entrada de la hacienda Trinidad o del camino a Lliulliu, había una distancia de 6,3 kilómetros. Se aseguró que los 


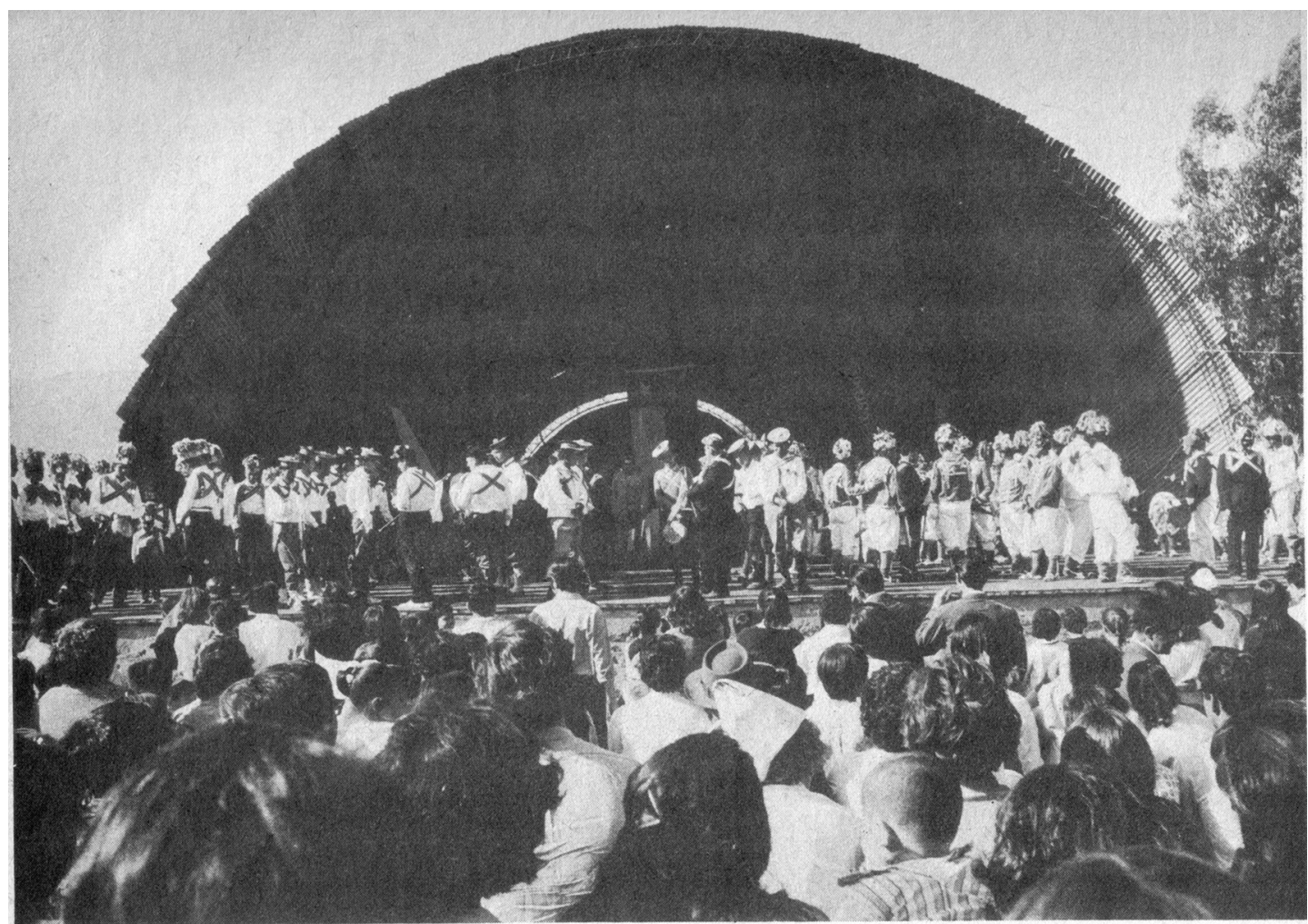

Fotografía 2. Corresponde al escenario de la Vaguada, en el que comienzan a tomar posición los conjuntos. Los bailes Chinos no fueron las únicas actividades que Hugo Arellano organizó en este escenario. En él se presentaron casi todos los artistas de la llamada "Nueva Ola": Sussy Vecki, Mauro Cass, Lucho Dimas y el "Pollo" Fuentes, entre otros. Además de la Nueva Canción Chilena, con artistas como Rolando Alarcón, Patricio Manns y "Chile Ríe y Canta”, de René Largo Farías. Fotografía de Rebeca Yáñez (Bello y Yáñez, 1968: 46).

alféreces aprovecharían la instancia del encuentro para formar la Asociación Nacional de Bailes Chinos, cuyo propósito sería resguardar "la autenticidad y riqueza folklórica de estas prácticas y obtener el reconocimiento de las autoridades" (Ídem).

En 1967 sabemos además que se dispuso premios a quien o quienes destacaran más como baile, alférez, coreografía, lejanía (distancia geográfica), al alférez más primerizo y al baile más numeroso. En esa ocasión se anunció como jurado a Adriana Carrizo, profesora e investigadora; Luis Villalobos, presidente de la Liga de Estudiantes (organización dependiente de la Logia Franklin Delano Roosevelt de Limache); representantes de la Biblioteca Nacional y de la Universidad de Chile especialmente invitados para la ocasión, además del ya mencionado Hugo Arellano (El Mercurio, Valparaíso, 10 y 12 de octubre de 1967).

La tercera versión de este encuentro, de octubre de 1968, es la mejor documentada, pues se conservaron hasta el presente registros fotográficos y sonoros. Lo novedoso es que el premio central de este encuentro fue aportado por El Mercurio, mientras los otros fueron colaboraciones del Instituto de Investigación y Difusión Folklórica de la Universidad de Chile, la Dirección General de Turismo, la Asociación de Microbuses de Limache, la Asociación de Ahorro y Préstamo Diego Portales, la empresa de buses Expresos Sol del Pacífico y Radio Limache. Arellano consiguió además que la municipalidad local diera su apoyo a la iniciativa por su "trascendencia turística para la región", mientras la Dirección General de Turismo comunicó que, en el calendario nacional oficial, el día 12 de octubre sería reservado como "Día del Folklore y costumbres autóctonas" en la ciudad de Limache. Se aseguró, con cierta exageración, que con ello los encuentros de bailes chinos de la Vaguada pasarían a constituirse "en la máxima representatividad en esta especialidad, conjuntamente con los Bailes de 
la Tirana y de Andacollo". Solo tres de los bailes que habían participado el año anterior se sumaron a esta tercera versión: El Granizo, Los Maitenes y Santa Cruz de Mayo de Tabolango; los otros no habían asistido, por lo menos en 1967: Puchuncaví, Cachagua, Pucalán, El Rincón, Ocoa, Palmilla, además de hermandades de Ovalle y Coquimbo. La actividad parece haber seguido una rutina similar a la de 1967, comenzando con el "recibimiento" en la estación de Limache, para desde allí dirigirse a la radio, "chineando", mientras avanzaban por las calles del pueblo. En esta ocasión, contamos con indicaciones específicas de cómo debían ser las presentaciones en la Vaguada: "cada baile efectúa sus 'flauteos' y 'mudanzas' durante diez minutos; el alférez tiene para sus 'cuartetas' o décimas siete minutos. Las diabladas presentes al estilo de ' $\mathrm{La}$ Tirana' actuarán separadamente". Cuando estuviese "actuando un baile en el escenario", los otros mantendrían silencio, "para que el público pueda captar los detalles de cada presentación y emitir posteriormente sus veredictos", medida que puede estar dando cuenta de que ello no era lo que ocurría necesariamente en la práctica (La Prensa, Limache, 4 de agosto y 9 de octubre de 1968; El Mercurio, Valparaíso, 6, 11 y 12 de octubre de 1968).

Siempre con relación a la tercera versión de este festival, se informa que había gran interés por presenciar el "espectáculo de autóctono folklore", por lo que Limache sería invadido de visitantes interesados en presenciarlo, ya que incluso desde el extranjero estaban solicitando antecedentes, "dada la originalidad del vestuario y del instrumental rústico que usan". La actuación en la Vaguada se programó desde las 14 horas, lugar hasta donde habría "locomoción especial desde y hasta la estación de ferrocarriles. Igualmente los taxibuses Sol del Pacífico mantendrán un servicio especial de Valparaíso hasta La Vaguada" (La Prensa, Limache, 4 de agosto y 9 de octubre de 1968; El Mercurio, 6, 11 y 12 de octubre de 1968).

La última versión de este festival se realizó en octubre de 1969. Se afirmó que el gran interés que despertó llevó a que la comisión organizadora se viese obligada a realizar una "delicada selección" entre los participantes, pues se trataba de una actividad que había sido aplaudida y admirada "por miles de personas de diversos lugares del país y especialmente por observadores extranjeros en años anteriores". Los grupos seleccionados para este encuentro fueron el baile del Niño Dios de las Palmas
(Quebrada de Alvarado), alférez Carlos Bernal; baile de Corpus Christi (Los Maitenes, Caicai), alférez Faustino Morales; baile Parroquial de Puchuncaví, alférez Guillermo Villalón; baile de la Quebrada (Hacienda de Cachagua), alférez Oscar Villalón; baile de Granizo (Olmué), alférez Luis Araos; baile de la Virgen del Carmen de Cabildo (Cabildo); Diabladas nortinas y baile de las Flechas Rojas. Se especifica que este cuerpo de "danzantes" actuaba sin alférez "a la usanza nortina", y que había sido seleccionado como el mejor baile en la última Fiesta de La Pampilla. Contaba con 45 integrantes que exhibían "una extraordinaria calidad coreográfica" (El Mercurio, Valparaíso, 12 de octubre de 1969; La Unión, Valparaíso, 12 de octubre de 1969; Revista 7 Días, octubre de 1968).

Para entonces los bailes chinos comenzaron a nombrase cada vez más seguido en diversos contextos y situaciones. Por ejemplo, en octubre de 1968 se presentaron en Olmué, en la cancha de la Asociación de Fútbol de esa comuna, para conmemorar el aniversario de los bailes de la Virgen de Lourdes y el Parroquial de Olmué, participando bailes de Petorquita, Boco, Hijuelas, Laureles, La Peña, Las Palmas, Caicai y Lo López (La Prensa, Limache, 21 de septiembre de 1968; El Mercurio, Valparaíso, 12 de octubre de 1968). A su vez, el mismo día de la presentación en el escenario de la Vaguada, uno de los bailes se estrenaría ya en la noche, en el Parque Municipal de Limache, donde continuaría la programación realizada por el Comité de Turismo local, que culminaría el 13 de octubre con "correduras y amansaduras en la Medialuna Municipal, con participación de los huasos del Club de Rodeos de Villa Alemana y Limache". Finalmente, el 22 de octubre de 1969 los bailes chinos se presentaron en la Feria Internacional de Santiago (FISA), para ello se nominó a los bailes de Los Maitenes, Granizo, Las Palmas y Cabildo (La Prensa, Limache, 22 de octubre de 1969).

\section{Canto a lo humano}

Como ya lo mencionamos antes, testigos privilegiados e informantes claves del tercer Encuentro de Bailes Chinos realizado en Limache fueron Enrique Bello y la fotógrafa Rebeca Yáñez. Su trabajo etnográfico más las transcripciones que hicieron de las presentaciones de los alféreces, cuyas grabaciones fueron cedidas por Hugo Arellano para facilitar su 
transcripción, permiten realizar un análisis más profundo de su significado.

En primer lugar, interesan las reflexiones que Bello realiza respecto de los alféreces. Observó que eran generalmente hombres mayores de sesenta o más años, a pesar que los rigores del trabajo en el campo, el estar curtidos por el sol, les hacían parecer tener mayor edad. Resalta su carácter de improvisadores de versos "lo más perfectos posibles", en décimas o cuartetas, que consistían en saludos, parabienes y augurios a su baile, a los oyentes y espectadores. Indica que les estaba prohibido ensayar o prepararse antes de su presentación. Piensa que la mayoría era analfabeto. Y se explaya:

"sale adelante con la pequeña bandera que va a servirle de 'cuerda' a su estro: la va enrollando a medida que improvisa, y cuando dice el último verso, la bandera ha quedado enrollada por completo" (Bello \& Yáñez, 1968: 49).

Insistiendo en su carácter improvisador indica que habiendo escuchado a muchos, era imposible dudar de esa cualidad, pues sacaban motivos del ambiente en el que se encontraban, relacionados con incidentes que ocurrían en el momento, así como también por la imperfección en la métrica octosilábica. Llamó la atención que no siempre estaban sobrios, pues "mientras esperan su turno, las libaciones van empañándole la visión, dañando su técnica..." (Ibídem: 50). Y agrega:

"Lo fabuloso es que en este encuentro de Limache comprobamos que algún alférez que apenas se sostenía en pie fue capaz, así y todo de improvisarse de un solo viaje, un saludo que completó diecinueve cuartetas y una décima, interpolando referencias a situaciones que ocurrieron mientras cantaba. Uno piensa, entonces, en lo que este poeta campesino pudo dar si su cabeza y sus piernas lo hubieran sostenido con las fuerzas normales" (Ídem).

Un último aspecto que Bello resalta de las improvisaciones de los alféreces, es que estas se realizan en circunstancias adversas para inspirarse:

“... a campo raso, bajo un sol abrasador, después de más o menos abundantes libaciones, en medio del bullicio multitudinario de los oyentes, y con algún sector del público en contra, ya que cada Baile acarrea hasta el lugar del encuentro a sus coterráneos, que harán coro a su alférez en desmedro de los cantores de otros conjuntos" (Ibídem: 51).

En segundo lugar, nuestro autor también realiza algunas reflexiones importantes sobre los danzantes. Destaca que estos "actúan con piernas y boca", además de la energía que despliegan. Que se trata de una danza masculina "de hombres de la montaña, duros y necesariamente exactos". Divaga que esta danza no llegó con los colonizadores sino que sus primeros cultores habrían sido los "serranos y montañeses precolombinos y hasta seguramente preincaicos". En relación con el atuendo, puntualiza que el correspondiente a las cofradías del Aconcagua y Valparaíso era menos ostentoso que en la Tirana:

"Los colores que aquí se ven más son el rojo, el blanco y el azul, seguramente por nacionalismo banderizante. Pero donde las diferencias son mayores es en el sombrero: gorros adornados con flores artificiales unos, más sencillos otros; sombreros con grandes floripondios de tiras plásticas, multicolores, espejos y espejuelos, objetos brilladores, colgajos atractivos de la luz. Los pies van calzados con botas rojas o simplemente gruesas medias de lanas rojas; sandalias o alpargatas, combinadas con medias de color que avanzan sobre la pantorrilla dejando caer un breve cordón" (Ibídem: 50-51).

En tercer lugar, Bello reflexiona sobre las décimas correspondientes al festival de octubre de 1968, de las cuales adelanta que,

"en el encuentro de Limache los Bailes Chinos de cada localidad presentaron cantos (saludos) a lo humano, a través de sus líricos alféreces, pues no era aquella una festividad religiosa, como las de su costumbre, sino que una civil, con contenidos de actualidad y no cultos adoratrices" (Ibídem: 51).

Lo anterior no significa que lo religioso estuviese totalmente ausente, sino que no era la 

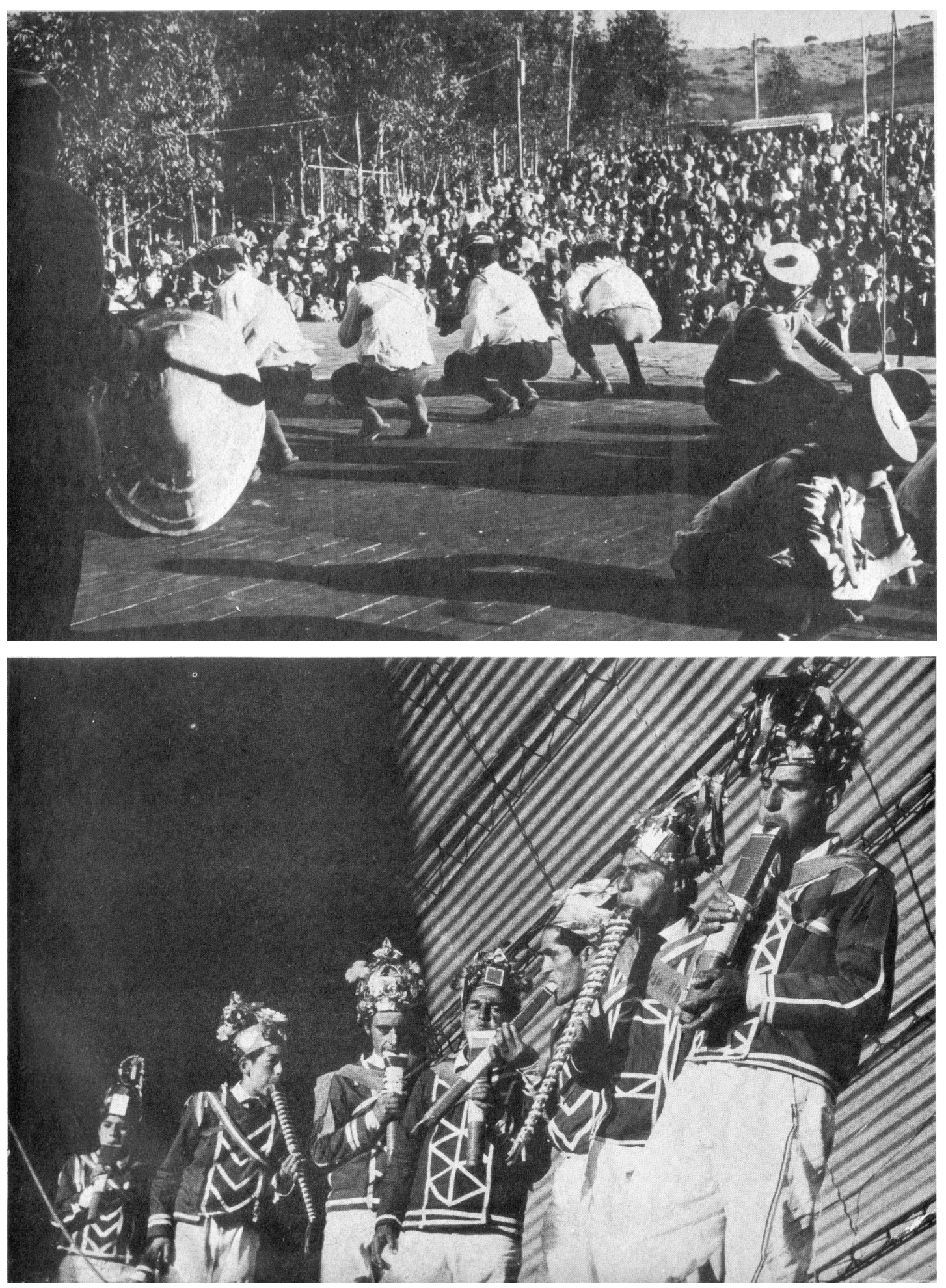

Fotografía 3. Arriba. Enrique Bello hizo el siguiente registro: "En estas danzas, los brazos no actúan: solo ocupan en tomar la flauta y golpear la percusión”. Fotografía 4. Abajo. Bello no especifica cuál es el baile en acción, solo indica que: "Las flautas son hechas de diversos materiales y formas. Un grupo ejecuta un sonido, otro le contesta encima; los dos golpes corresponden al que ejecutan los danzantes”. Fotografías de Rebeca Yáñez (Bello y Yáñez, 1968: 48-54). 
temática central de estas presentaciones. Incluso Faustino Morales, que era el alférez más devoto, hizo cuartetas con otros énfasis:

"Pa cantar no soy divino/se los puedo asegurar/y si encuentran algo mal/cárguelo a los limachinos//Que por poder divino/ yo he llegado hasta aquí/a este respetable público/a todos les digo así. /No canto del génesis/la historia me sofoca/cantaré literatura/porque la intención es poca" (Ibídem: 52).

¿Cuáles son las temáticas a las que se refirieron los alféreces? En primer lugar, destacamos los saludos iniciales, los que son de preferencia para el pueblo o público en general (presente en la actividad o como radioescuchas). Por ejemplo, el ya mencionado alférez Faustino Morales comenzó cantando la siguiente décima:

"Al pueblo en primer lugar/y con mucha devoción/al pueblo en primer lugar/le debo esta devoción, /yo los quiero saludar/con toda mi hermanación, /yo les brindo esta atención/y a su presencia me tienen/reciban saludación/del Baile de Los Maitenes".

Oscar Villalón por su parte comenzaría diciendo: "Respetao público/se lo digo atentamente/ le rindo las buenas tardes/ay, a todos los oyentes" (Ibídem: 52, 54).

El alférez Carlos Bernales, cantando por el baile de Granizo, señaló:

"Para empezar a cantar/para empezar a cantar/permiso pido primero/y a don Hugo Arellano, /señorita y caballero. /Para cantar soy sincero/con la bandera en la mano/y al público presente/y al público presente, /al moderno y al anciano" (Ibídem: 56).

Hugo Arellano, impulsor de la actividad, es nombrado como autoridad pero más bien como una persona cercana. El alférez Óscar Villalón, del baile de Puchuncaví, por ejemplo, señaló: "Aquí le canto a don Hugo/lo digo aunque me emborrache/cantándole unos versitos/a esta radio de Limache". Por su parte Arturo Ogaz declaró: "Me da gusto el recordar/y unidos por este mate /lo que está haciendo don Hugo/aquí en la Radio Limache" (Ibídem: 59).

Con relación al saludo que se realizan entre alféreces, se dirigen a quienes ofician de anfitriones por corresponder a bailes de la comarca o bien, en el caso de los "dueños de casa" a algunas de las cofradías que van de visita. Puede haber también un discurso oculto, en el sentido de lo planteado por James Scott, en que públicamente se manifieste adhesión y cercanía, aunque también estuviese presente el afán de competir, si bien había suficientes premios para todos (Scott, 2003: 22 y ss.). Así Morales, dejando el paso al baile de Puchuncaví señaló: "Le digo estos parabienes/esto a mí me conforta/voy a cederle el lugar/a los amigos e' la costa", pues como había dicho antes, a los bailes visitantes "no hay que atacar". Villalón manifestó: "Y del baile de los Maitenes/la geruza se me encierra,/es muy grande la amistad/y venimos a esta tierra"; agregando más adelante:

"Esta cosa "e los costinos/lo digo y no soy profano/porque al pueblo de Limache/lo queremos como hermano. //Al amigo Lucho Araos/haré un versito y refale/soy amigo de don Lucho/y con Faustino Morales".

Oscar Villalón de la Quebrada de Puchuncaví, por su parte respondía a su turno:
"Amigo Faustino Morales/pa todo pongo cuidao/y especialmente me invitó/el amigo Luis Araos/el amigo Luis Araos //El amigo Luis Araos/me pidió este favor, /yo he lle- gado a esta Radio/por hacer cooperación/ por hacer cooperación” (Ibídem: 52-54).

Un segundo aspecto que destacó en las presentaciones de octubre de 1968 fueron las valoraciones positivas que se hicieron de la radio como medio de comunicación, en contraposición a la idea que estos solo fueron un espacio de control social estatal. La improvisación de Arturo Ogaz, del baile de la Cruz de Mayo de Tabolango, es la más elocuente de todas (Ibídem: 55):

"A las radios de la zona / voy a rendir homenaje/artistas y personajes/que escuchan a esta hora,/a todas las auditoras/ de este escenario saludo,/contando muy a menudo/digo: señores y señoras/para 
Chile y el escudo/trabajan las emisoras.// Esta es la Radio Limache,/la que organiza esta fiesta/y don Hugo se presenta/pero con gran personal,/lo mismo que en un mensaje/que aquí en tierra limachina/que con su mano divina/el hombre planta el tomate,/y este año tuvo un desastre/con la maldita sequía... //La Radio Valparaíso,/ Portales, Cooperativa,/ primera vez en mi vida/que estos versos le improviso,/ellos nos dan el aviso/y nos tienen informados/ de todo lo que ha pasado/en el momento preciso...//La Radio nos pone alegres/en un baile familiar,/también se puee buscar/a alguno de sus parientes/con el trasmisor al frente./A veces siento pesar:/sangre de algún hospital/piden para algún paciente,/ van los dadores al frente/para esta vida salvar.//Es el deber de la Radio/mantener en general,/todos cumplen sin desaire/en el día su sonar./Corresponsales son varios/ en Chile y el extranjero,/parecen grandes pioneros./Yo quiero mejor cantar./Estoy en este escenario/de aquí, pidiendo por ellos./ Todo lo cumplen muy bien/para con toda la nación,/con signos de admiración/yo quiero hacerlo saber $/ i$ La radio lo informa a usted!/a nuestro Chile ofendido...// También la radio nos cuenta/de la ciencia de este mundo./Inmorales e inmundos/ también los da a conocer./ Parlamentarios también/a la radio se presentan/y en sus discursos nos cuentan/sus programas por hacer,/y para hacernos creer/cualquier mentira inventan.//Limache, Viña del Mar,/ Recreo con Minería,/También la Radio Reloj/Junto a la Cooperativa/(improviso en este día)/Portales y Valparaíso/Agricultura preciso/y aquí estoy con alegría/¡Viva mi patria querida/que Chile es un paraíso!".

El alférez Arturo Ogaz hace una valoración completa del rol de la radio: informa, entretiene, alegra y entrega servicios de utilidad pública. También era espacio para los políticos embaucadores. Importante la crítica, porque para la clase política de ese entonces -y para ciertos especialistas-, los campesinos eran "ganado" manipulable, pero este testimonio demuestra que en los sectores rurales se conocía bien la dinámica de la política urbana, y que por tanto, cuando aceptaban ir a sufragar por medio de un acarreo, era por su propia voluntad pero, probablemente, a partir de lecturas específicas de relación costo-beneficio. En la misma línea de lo que comentamos, destacables fueron los versos improvisados por Carlos Bernales, quien reconoce además el rol social de la radio: "Por otro que está por verse/esta es la Radio e' los pobres,/cuando yo pongo un aviso/cuando yo pongo un aviso,/no me cuesta ningún cobre..." (Ibídem: 56-57). Por su parte, el alférez Luis Araos resalta el hecho que gracias a los encuentros organizados y a su transmisión, los bailes chinos se habían dado a conocer:

\begin{abstract}
"De los grandiosos Bailes Chinos/cantando así lo digo/que en regiones apartadas/que en regiones apartadas /esto estaba escondido...//Cantando mi voz sea dicha/y gracias a esta Radio/demos gracias a esta Radio/ que ha salido a la vista/que ha salido a la vista" (Ibídem: 58).
\end{abstract}

Un tercer aspecto que se aprecia con nitidez en las recitaciones de los alféreces, son los relacionados con sus actividades económicas y de manera más puntual, la dura sequía de la segunda mitad de la década de 1960, que los estaba afectando dramáticamente. Ya habíamos citado las décimas de Arturo Ogaz, cuando en una parte de ellas señala: "Que aquí en tierra limachina/que con su mano divina/el hombre planta el tomate,/y este año tuvo un desastre/con la maldita sequía" (Bello \& Yáñez, 1968:59). Quien desarrolla más extensamente esta problemática es Alfredo Villalón, alférez del baile de Puchuncaví:

"Este es el baile costino,/para mí no habrán clamores/es un baile campesino/y somos agricultores... //Por eso lo digo aquí,/con mi bandera florida,/voy a iniciar unos versos/ agregaos a la sequía.//Para esta gran sequía/ así mi voz lo dirá/la costa e' Puchuncaví/se encuentra muy afectá.//Se encuentra muy afectá,/si hasta mi mente se arrisca,/a sus padres ya muy tristes/reclaman sus pobres hijas.//Para los agricultores/esto ya no es muy florido/el pueblo de Puchuncaví,/ hasta la semilla ha perdido.//La semilla ha perdido/lo digo y así es mi guía,/se perdió pues la semilla/por culpa de la sequía.//El sacrificio del agricultor/aquí se lo explico yo,/lo que trabajó en muchos años/ahora 

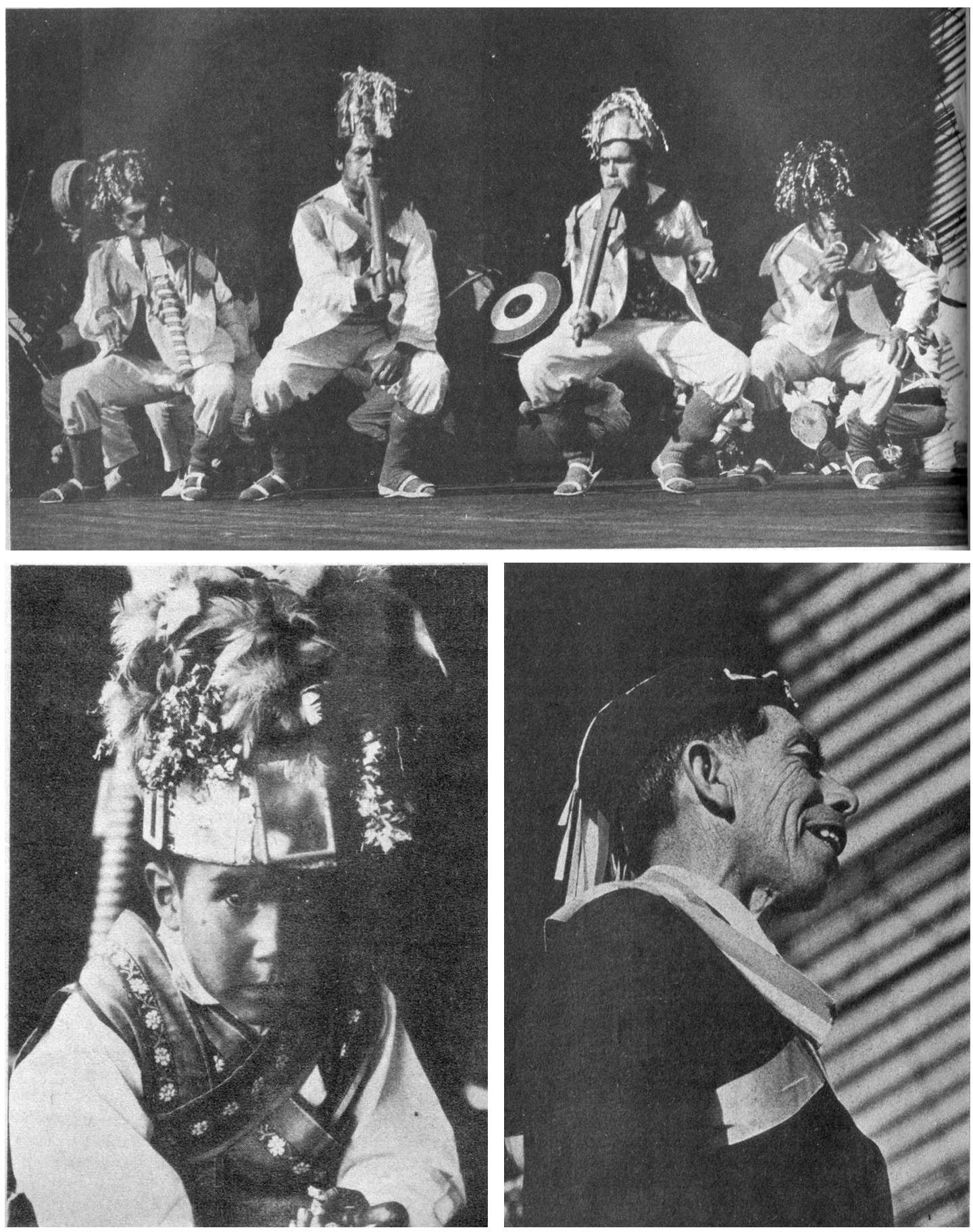

Fotografía 5. Arriba. En este caso Enrique Bello hace notar la factura de la pifilca, además de indicar que se toman con una mano, mientras la otra se deja libre para lograr "una mayor perfección del movimiento muscular de los danzantes". Fotografía 6. Abajo izquierda, niño con significativo atuendo, que "semeja un Niño-Dios de iglesita aldeana, para quien él está siempre dispuesto a bailar". Fotografía 7. Abajo derecha, Faustino Morales, sucesivamente elegido mejor alférez en los festivales de bailes chinos. (Bello y Yañez, 1968: 47,52,60). 
todito lo perdió.//Ahora todito lo perdió/lo digo y no me desdigo/que el señor de los cielos/para todos dio el castigo".

La temática de la sequía estuvo presente permanentemente en los cantos de los alféreces, representantes de comunidades de campesinos o pescadores. Villalón explica los efectos de la sequía, la pérdida del trabajo de años y las consecuencias económicas y sociales a nivel familiar. Interesante es que si bien se hace referencia a que todo se debió a un castigo divino, no se realiza ninguna invocación o solicitud para que ello sea revertido ni a Dios ni a la Virgen, como es el caso del canto de Aurelio Frez a la Virgen del Carmen de Pachacamita de 1955 (Mercado \& Rondón \& Piwonca, 2003: 45). Aunque en este caso la problemática de la sequía no se asocia a responsabilidades políticas, se pueden hacer conexiones con los análisis que respecto de los bailes de Puchuncaví y en un contexto de crisis ambiental han realizado Francisco Sabatini y Francisco Mena. Desde su punto de vista, a pesar que treinta años de emanaciones de gases nocivos para la salud y la agricultura parecían no haber motivado a la población a movilizarse y a cuestionar la situación, los puchuncavinos sí mostrarían "una considerable capacidad de organización y activismo, cuyo punto más alto se alcanza durante las festividades religiosas, con los célebres bailes 'chinos' de Puchuncaví. Su despliegue de colorido, danzas y devoción es la cara visible de una extensa y compleja red de relaciones y trabajo que hunde sus raíces en la sociedad local". Subrayan que además de capacidad de acción, los bailes evidencian

\footnotetext{
"una faceta práctica de conexión con los problemas cotidianos de la gente. Lejos de ser una simple forma de escapismo, las rogativas que estructuran el baile se refieren en forma directa y hasta ingenua a las cuestiones que amenazan su sobrevivencia terrenal: enfermedades, falta de trabajo y sequías, entre las principales" (Sabatini \& Mena 1995: 51-52).
}

En el caso que estamos analizando no tienen el carácter de rogativas pero sí muestran conciencia crítica, con cierta cuota de resignación y fatalismo, lo que no creemos que se deba a una lógica de la religiosidad popular sino simplemente a un análisis realista de la situación.
Un cuarto aspecto al que se refieren los alféreces es a la contingencia urbana, asociada a las noticias importantes a las que sin duda accedieron por medio de la misma radio o los diarios. Ciertamente que la cultura campesina descansaba en la oralidad, pero en este punto Enrique Bello se equivocaba. Los alféreces no eran analfabetos. Eran buenos lectores, especialmente de la Biblia o de textos religiosos, que en algunos casos puntuales les eran cedidos por sacerdotes y en otros por alféreces más viejos que asumían el rol de maestros, o lisa y llanamente, por familiares (Entrevista a Faustino Morales 2002). Sin embargo, las décimas cantadas en el escenario de la Vaguada dan cuenta además, como ya ha quedado claro, que escuchaban la radio y leían los diarios. Un ejemplo ilustrativo de lo que señalamos lo constituyen las décimas recitadas por el alférez Carlos Bernales, referidas a la primera operación de trasplante de corazón, realizado el 28 de junio de 1968, por el equipo médico encabezado por el doctor Jorge Kaplán, a María Elena Peñaloza, joven de 24 años, con el corazón de Gabriel Véliz, de 21 años, fallecido de un tumor cerebral, cirugía realizada en el Hospital Naval de Valparaíso. Esta noticia tuvo un significativo impacto social, pues trasplantar un corazón era definitivamente ganarle a la muerte. Canta este poeta campesino:

"Para pasar a otro punto,/parece que fuera un sueño,/para hacerles recordar/a los doctores porteños.//En Chile ha sido el primero/el doctor Jorge Kaplán/que su fama ha llevar/y por todo el mundo entero/ en los países extranjeros/como en el país chileno/donde hay doctores tan buenos/ que al hombre antes de morir/ay, lo hacen revivir/con un corazón ajeno.//Quisiera ser bien sereno/para poderme expresar /y estos versos cantar/aquí y en cualquier terreno,/y estos doctores tan buenos/los del Hospital $\mathrm{Naval} /$ luchan por salvar enfermos/y su vida prolongar/llevando felicidades/a todos los del lugar" (Ibídem: 56-57).

Poco antes que se realizara el tercer encuentro, en la Radio Limache, el alférez del baile de la Santa Cruz de Mayo de Tabolango, Arturo Ogaz, también realizó un canto improvisado sobre el trasplante de corazón realizado a María Elena Peñaloza (Ibídem: 59).

Un último aspecto que es tratado por los alféreces, aunque de manera periférica, es la efeméride 
con la que Hugo Arellano convocó a estos festivales de bailes chinos. Nos referimos al 12 de Octubre. A la sazón no se aprecia todavía una mirada crítica. Las efemérides por mucho tiempo fueron parte del currículum escolar y había una cultura asociada a ellas. Así por ejemplo, independientemente de los discursos ocultos, que hubiese "carreristas" y "o'higginistas" por ejemplo, en todas las instituciones públicas se hacían actos en que se conmemoraba la figura de O'Higgins como "libertador". Larraín ha estudiado cómo a partir de la dictadura se produjeron rupturas que llevaron a que ciertos próceres dejaran de valorarse como representativos de la nación pasando a ser representativos "del otro" con el que se levantaron diferencias irreconciliables (Larraín, 2010, 22-24). En el caso que estamos estudiando, fue valorado el espacio que se abrió para poder presentarse, pero prácticamente no hay alusiones al "Día de la Raza". Una de ellas es de Oscar Villalón, que además realiza una de las escasas referencias que encontramos a lo religioso:

"Por hacer cooperación/mi garganta se me tupe/porque voy a celebrar/este doce de octubre/este doce de octubre.//Porque es 12 de octubre/lo digo y lo que me pasa/tuvimos que celebrar/y el día de la Raza. //Por el Día de la Raza/por el libro de Jacob,/cuando Cristo sacó al luz/la América descubrió./ La América descubrió/ay lo digo y muy pronto/y esto fue celebrado/en la Iglesia el 3 de agosto" (Bello \& Yañez, 1968: 54).

Da la impresión que el alférez Villalón parece haber buscado más la rima que querer expresar una idea concreta respecto del concepto de raza, como popularmente se entendía en la época.

\section{Reflexiones finales}

En el contexto de la creciente valoración de la cultura popular a partir de la segunda mitad del siglo $\mathrm{XX}$, se aprecia que los bailes chinos, que se han visualizado hasta ahora esencialmente como expresión de la fe del pueblo, por medio de su palabra, también dieron cuenta de "lo humano", es decir, de las problemáticas que estaban afectando a sus comunidades. El espacio que abrió Hugo Arellano, en la Vaguada de la Radio Limache, fue uno de los canales por donde la cultura de los sectores populares de raíz campesina comenzó a fluir en los espacios urbanos, permitiendo su reconocimiento a una escala más amplia en términos sociales, como también por los círculos académicos. Los contenidos que se expresan en el "canto a lo humano" de los alféreces, dan cuenta de lo importante que estaba siendo la radio como medio de comunicación, pero también de las preocupaciones y mentalidad campesina de aquel entonces, que distaba de estar ajena a la contingencia, como de tener una opinión superficial sobre la misma. Si contrastamos los contenidos de los cantos de los alféreces de la presentación de octubre de 1968 con las que se hacían en las fiestas religiosas de Valparaíso o Aconcagua, la principal diferencia está en el carácter devocional de las segundas, mientras las primeras tienen un acento en donde se da cuenta de los problemas que afectan a campesinos, como fue el caso de la sequía mencionado acá. En ese sentido, los cantos de los alféreces en las presentaciones de la cofradías en el escenario de la Vaguada de la Radio Limache quizá son, por su contenido humano y social, más comparables con la poesía de la Lira popular, que como bien lo sintetiza Patricio Manns, se referían, en décimas, a las noticias de la actualidad nacional y extranjera (Manns, 1986: 52-53).

Lo anterior refuerza la idea que la religiosidad popular no viene a ser más que un vértice de una cultura popular, que en sí misma es a la vez compleja, diversa y versátil; siendo todavía un desafío conocerla y comprenderla en profundidad. A su vez, resulta evidente que la radiodifusión, por lo menos para la década de 1960, era bastante más que un instrumento de control social del Estado, siendo una valiosa instancia de interrelación e identificación comunitaria y de proyección de lo local en lo global. La relación inversa ya ha sido probada.

\section{Agradecimientos}

Agradezco las observaciones de Rafael Contreras, Daniel González y la revisión de diarios realizada por Esteban Cisternas. También las transcripciones de entrevistas por los profesores Esteban Faúndez, Esteban Briceño, Manuel Ávila y Carolina Vidal. Este proyecto es resultado del Fondart Nacional 2014, N 38.707: "Fiestas de bailes chinos del norte grande, el norte chico y la zona central. Una descripción antropológica e histórica de su patrimonio". Se agradecen igualmente las valiosas observaciones de los evaluadores. 


\section{Referencias Citadas}

Bauer, A.

1994 La Sociedad Rural Chilena. Desde la Conquista Española a nuestros días. Editorial Andrés Bello, Santiago de Chile.

Bajtin, M.

1989 La cultura popular en la Edad Media y en el Renacimiento. El contexto de François Rabelais. Editorial Alianza, Madrid, España.

Burke, $\mathrm{P}$.

1991 La cultura popular en la Europa moderna. Editorial Alianza, Madrid.

Borde, J. \& Góngora, M.

1956 Evolución de la propiedad en el valle de Puangue (vols. 1-2). Editorial Universitaria, Santiago de Chile.

Brucher, M.

1962 Estudio socioeconómico de la comunidad agrícola de Caleu. Tesis Ingeniero Agrónomo, Universidad de Chile, Santiago de Chile.

Chavarría, P.

2009 "De los cogollos del viento". Los saberes de los antiguos. Ed. Archivo Literatura Oral de la Biblioteca Nacional \& Dirección de Bibliotecas Archivos y Museos, Concepción, Chile.

Chavarría, P.

2015 La Guitarra es la que Alegra. Editorial Cuarto Propio, Concepción, Chile.

Chartier, R.

1995 Sociedad y Escritura en la Edad Moderna. Ed. Instituto Mora, México.

Donoso, K.

2009 "Por el arte-vida del pueblo: Debates en torno al folclore en Chile. 1973-1990". Revista Musical Chilena, vol. 63, $\mathrm{N}^{\circ}$ 212: pp. 29-50.

Contreras, R. \& González, D.

2009 Este baile de Cay Cay. Editado por Etnomedia, Santiago de Chile.

Contreras, R.; González, D. y Pineda, M.

2014 Bailes chinos de Chile central (1968-1969). Etnomedia, 74 minutos, recurso sonoro, Santiago de Chile.

Contreras, R.; González, D. y Pineda, M.

2014 Comunidad Las Palmas (1968-1970). Canto, rito y territorio. Etnomedia, 74 minutos, recurso sonoro, Santiago de Chile.

Contreras M., R. \& González, D.

2015 Bailes Chinos, Festividades y Religiosidad Popular en el Norte Chico. Consejo Nacional de la Cultura y de las Artes, Santiago de Chile.

Dannemann, M.

1998 Enciclopedia del Folclore de Chile. Editorial Universitaria, Santiago de Chile.

González, D.; Contreras, R. y Pineda, M.

2014 Canto campesino de Chile central (1962-1971).

Etnomedia, 74 minutos, recurso sonoro, Santiago de Chile.

Godoy, M.

2007 Chinos. Mineros-danzantes del Norte Chico, siglos XIX $y X X$. Consejo Nacional del Libro y la Lectura, Santiago de Chile.

Larraín, J.

2010 "La identidad Chilena y el Bicentenario". Estudios Públicos $\mathrm{N}^{\circ} 120$, pp. 5-30.
Larkin, B.

2008 Signal and Noise: Media, Infrastructure, and Urban Culture in Nigeria. Duke UP, Durham y Londres.

Manns, P.

1986 Violeta Parra. La guitarra indócil. Ediciones Literatura Americana Reunida, Concepción, Chile.

Martí i Pérez, J.

1990 "El folklorismo. Análisis de una tradición "pret-a-porter", Revista de Musicología del CSIC, $\mathrm{N}^{\circ}$ 45, pp. 317-352.

Martí i Pérez, J.

1999 "La tradición evocada: folklore y folklorismo". Citado de: http://hdl.handle.net/10261/38658

Mercado, Claudio

1995 "Permanencia y cambio en fiestas rituales de Chile Central”, Revista Valles, I/1, pp. 11-29.

Mercado, Claudio

1995-1996 "Música y estados de conciencia en fiestas rituales de Chile central. Inmenso puente al universo". Revista Chilena de Antropología, No 13, pp. 163-196.

Mercado, Claudio

2002 "Ritualidades en conflicto: los bailes chinos y la Iglesia Católica en Chile Central". Revista Musical Chilena, v. 56, $\mathrm{N}^{\mathrm{o}}$ 197, pp. 39-76.

Mercado, C.; Rondón, V.; Piwonca, N.

2003 Con mi humilde devoción. Bailes Chinos en Chile Central. Editado por Museo de Arte Precolombino, Santiago de Chile.

Mercado Guerra, J.

2014 "Práctica ritual y tensiones identitarias en las danzas promesantes de la fiesta del Santuario de Ayquina, norte de Chile”. Revista Diálogo Andino, No 45, pp. 193-213.

Ornelas, R.

2014 "Radio y cotidianidad en México. 1900-1930", en Aurelio de los Reyes (Coordinador), Historia de la vida cotidiana en México. Tomo V: vol. 1. Siglo XX. Campo y ciudad, El Colegio de México, México, pp. 127-169.

Pérez de Arce, J.

2017 "Bailes Chinos y su identidad invisible", Chungara (Arica), vol. $49 \mathrm{~N}^{\circ} 3$ set. 2017, pp. 427-443.

Ruiz, A.

1995 "Hegemonía y marginalidad en la religiosidad popular chilena: los bailes ceremoniales de la región de Valparaíso y su relación con la Iglesia Católica”, Revista Musical Chilena, Año XLIX, Julio-Diciembre, No 184, pp. 65-83.

Sabatini, F. \& Mena, F.

1995 "Las chimeneas y los bailes 'chinos' de Puchuncaví". Ambiente y Desarrollo, vol. XI, N³, 1995, pp. 52-59.

Salinas, M.

1991 Canto a lo divino y religión popular en Chile hacia 1900. Edic. Rehue, Santiago de Chile.

Sepúlveda, F.

2009 El canto a lo poeta. A lo divino y a lo humano. Análisis estético antropológico y antología fundamental. Editorial Universitaria, Santiago de Chile.

Scott, J.

2003 Los dominados y el arte de la resistencia. Editorial Txalaparta, México.

Subercaseaux, B.

2011 Historia de las ideas y de la cultura en Chile, vol. III. Editorial Universitaria, Santiago de Chile. 
Torres, R.

2004 "Cantar la diferencia. Violeta Parra y la canción chilena", Revista Musical Chilena, $\mathrm{N}^{\circ} 201$, pp. 53-73.

Uribe, J.

1958 Contrapunto de alféreces en la Provincia de Valparaíso. Ediciones Anales de la Universidad de Chile, Santiago de Chile.

Venegas, $\mathrm{F}$

2009 Los herederos de Mariana Osorio. Comunidades Mestizas de Olmué: Repúblicas campesinas en los confines del Aconcagua Inferior, ss. XVII-XXI. Ediciones Carjana, Santiago de Chile.

Venegas E., F.

2012 "Una mirada a las reflexiones sobre los mestizos y los mestizajes", en Concepción y el Bicentenario. Miradas a su Historia Republicana, editado por A. Bancalari \& M. Rojas. \& M. Valdés \& J. Ventura, pp. 169-210. Editado por Departamento de Ciencias Históricas y Sociales, Concepción, Chile.

Venegas, F.

2014 Del asociacionismo rural a la asociatividad urbana. Protagonismo de la sociedad en la construcción de un espacio local. Tesis de Doctorado, Universidad de Chile, Santiago de Chile.

Venegas, F.

2017 Violeta Parra en Concepción y la Frontera del Biobío: 1957-1960. Recopilación, difusión del folklore y desborde creativo. Editado por Universidad de Concepción, Cátedra Violeta Parra y VRIM UdeC, Concepción, Chile.

Villalobos, Pablo

2012 "Prácticas y sentido de juego: relaciones entre la Iglesia Católica y los Bailes Chinos en las fiestas religiosas de la Región de Valparaíso, Chile”, Doble Vínculo, Pontificia Universidad Católica de Chile.

\section{Fuentes}

Consultadas en Biblioteca Nacional de Santiago de Chile:

- El Mercurio, 1966-1970

- La Unión, 1966-1970

- El Siglo, 1966-1970

- Revista 7 Días, 1966-1970.

Consultadas en Hemeroteca Biblioteca Luis David Cruz Ocampo, Universidad de Concepción:

- "Enrique Bello y Rebeca Yañez, Los Poetas de los bailes chinos de Limache cantan a lo humano", Revista de Educación, Ministerio de Educación No 11 , Santiago de Chile, octubre 1968.

- (Dirección de Estadísticas y Censos, 1960).

Entrevistas realizadas por Fernando Venegas E.

- Faustino Morales, Los Maitenes, 2002.

- Hugo Arellano, Limache, 2010. 
